This item was submitted to Loughborough's Research Repository by the author.

Items in Figshare are protected by copyright, with all rights reserved, unless otherwise indicated.

\title{
Contrast-enhanced spectral mammography improves diagnostic accuracy in the symptomatic setting
}

\section{PLEASE CITE THE PUBLISHED VERSION}

http://dx.doi.org/10.1016/j.crad.2016.05.009

\section{PUBLISHER}

(C) The Royal College of Radiologists. Published by Elsevier

\section{VERSION}

AM (Accepted Manuscript)

\section{PUBLISHER STATEMENT}

This work is made available according to the conditions of the Creative Commons Attribution-NonCommercialNoDerivatives 4.0 International (CC BY-NC-ND 4.0) licence. Full details of this licence are available at: https://creativecommons.org/licenses/by-nc-nd/4.0/

\section{LICENCE}

CC BY-NC-ND 4.0

\section{REPOSITORY RECORD}

Tennant, Sarah L., Jonathan James, Eleanor J. Cornford, Yan Chen, Helen C. Burrell, Lisa J. Hamilton, and Constantine Girio-Fragoulakis. 2019. "Contrast-enhanced Spectral Mammography Improves Diagnostic Accuracy in the Symptomatic Setting". figshare. https://hdl.handle.net/2134/21481. 


\section{Contrast-Enhanced Spectral Mammography Improves Diagnostic Accuracy in the Symptomatic Setting}

\section{Sarah L Tennant ${ }^{1}$, Jonathan J James ${ }^{1}$, Eleanor C Cornford ${ }^{1}$, Helen C Burrell ${ }^{1}$, Lisa J Hamilton ${ }^{1}$, Constantine Girio-Fragoulakis ${ }^{1}$, Yan Chen ${ }^{2}$}

1. Nottingham Breast Institute, Nottingham University Hospitals, Nottingham, UK

2. Applied Vision Research Centre, Loughborough University, Loughborough, UK.

Corresponding Author:

Sarah L Tennant

Nottingham Breast Institute, Nottingham University Hospitals NHS Trust, NG5 1PB

Tel: 0115 9691169, ext. 59022

Email: sarah.tennant@nuh.nhs.uk

Acknowledgements: With thanks to Mrs. Laura Willoughby (Education Administrator) for data entry and support.

1. Guarantor of integrity of entire study: Sarah L Tennant

2. Study Concepts and Design: Sarah L Tennant, Jonathan J James, Eleanor C Cornford

3. Literature Research: Sarah L Tennant, Jonathan J James

4. Clinical Studies: N/A

5. Experimental studies/data analysis: Sarah L Tennant, Jonathan J James, Eleanor C Cornford, Helen C Burrell, Lisa J Hamilton, Constantine Girio-Fragoulakis, Yan Chen

6. Statistical analysis: Yan Chen, Sarah L Tennant

7. Manuscript Preparation: Sarah L Tennant, Jonathan J James, Eleanor C Cornford

8. Manuscript Editing: Sarah L Tennant, Jonathan J James, Eleanor C Cornford 


\section{Abstract}

\section{Aim}

To assess the diagnostic accuracy of Contrast Enhanced Spectral Mammography (CESM), and gauge its "added value" in the symptomatic setting.

\section{Methods}

At our institution, women aged 35-70 years with a suspicious or malignant clinical abnormality are offered CESM instead of standard Full-Field Digital Mammography (FFDM) as an initial imaging test. CESM is also offered to younger women whose ultrasound is suspicious, or who have biopsy-proven malignancy. It is occasionally used as an alternative to breast MRI following multi-disciplinary team discussion.

We performed a retrospective multi-reader review of 100 consecutive CESM examinations. Anonymised Low Energy (LE) images were reviewed and given a score for malignancy. At least 3 weeks later, the entire examination (LE and recombined images) was reviewed. Pathology data was obtained for all cases. Differences in performance were assessed using receiver operative characteristic (ROC) analysis. Sensitivity, specificity and lesion size (vs MRI or histopathology) differences were calculated.

\section{Results}

$73 \%$ cases were malignant at final histology, $27 \%$ were benign following standard triple assessment. ROC analysis showed improved overall performance of CESM over LE alone, with area under the curve of $93 \%$ vs $83 \%(p<0.025)$. CESM showed increased sensitivity ( $95 \%$ vs $84, p<0.025)$ and specificity $(81 \%$ vs $63 \%, p<0.025)$ compared to LE alone, with all 5 readers showing improved accuracy. Tumour size estimation at CESM was significantly more accurate than LE alone, the latter tending to undersize lesions. In $75 \%$ of cases CESM was deemed a useful or significant aid to diagnosis.

\section{Conclusion}

CESM provides immediately available, clinically useful information in the symptomatic clinic in patients with suspicious palpable abnormalities. Radiologist sensitivity, specificity and size accuracy for breast cancer detection and staging are all improved using CESM as the primary mammographic investigation. 


\section{Contrast-Enhanced Spectral Mammography Improves Diagnostic Accuracy in the Symptomatic Setting}

\section{Introduction}

The diagnostic assessment of patients with breast symptoms is based on the multidisciplinary triple diagnostic method. This includes clinical assessment, imaging assessment and (where appropriate) needle biopsy (1). Mammography is the initial investigation of choice in women over the age of forty, and is also recommended in the assessment of younger women if clinically suspicious or malignant.

Mammography has its limitations, particularly in the younger woman, with denser breasts, where sensitivity is reduced (2). Contrast Enhanced Mammography (CESM) is a novel technique combining the benefits of iodinated contrast with standard mammographic views. Dual energy acquisitions during one mammographic exposure yield two sets of images a Low Energy (LE) set, equivalent to standard Full Field Digital Mammography (FFDM) (3) (4) (5) and a recombined set displaying contrast uptake. Despite encouraging data from Europe and North America demonstrating increased sensitivity and specificity of the technique over FFDM (3) (6) (7), there is only limited data to support its routine use as a tool in the diagnosis and staging of breast cancer.

Accurate local staging of breast cancer is important for treatment planning and prognostication. With the increase in availability of oncoplastic techniques, the demonstration of multifocal or multicentric disease means that patients previously advised to undergo mastectomy may now be offered breast conserving surgery. Larger tumours, previously warranting mastectomy, may be suitable for breast conserving surgery post neoadjuvant chemotherapy (NAC). Knowing the size and location of tumour foci aids surgical planning.

The aim of this study was to assess the diagnostic accuracy of CESM, and to assess its "added value" in the symptomatic setting. 
Since November 2013, CESM has routinely been performed in the symptomatic breast service at our institution, according to local departmental guidelines.

All patients seen in our one-stop symptomatic clinics are examined by a surgeon or specialist nurse practitioner, prior to undergoing any imaging. The clinical examination is graded as P1 - Normal, P2 - Benign, P3 - Indeterminate, P4 - Suspicious, and P5 - Malignant (1). Women aged 35-70 years with a suspicious or malignant (P4 or P5) clinical abnormality are offered CESM instead of FFDM as an initial imaging test. CESM is also offered to younger women whose ultrasound is suspicious, or who have biopsy-proven malignancy. It is occasionally used as an alternative to breast MRI, for example if the patient is claustrophobic, following multi-disciplinary team (MDT) discussion.

Renal function is not routinely available for patients attending symptomatic breast clinic, so we have taken a pragmatic approach and do not perform CESM in patients at increased risk of renal impairment. This includes patients age $>70$ years, diabetics and anyone with a history of renal disease. Patients with previous reaction to iodinated contrast agents, known iodine allergy and patients who are pregnant or lactating are also excluded from CESM.

The CESM examination was performed on a commercially available system (Senobright, GE Healthcare). All patients undergoing CESM gave written consent for the procedure.

In order to assess the diagnostic accuracy of CESM in the symptomatic setting a retrospective, multi-reader study was undertaken of our first 100 consecutive CESM examinations comparing LE images alone, to the entire CESM examination (i.e. LE and recombined images). Ethics approval was not deemed necessary, following discussion with our local Research and Development Team as this retrospective review was considered to represent audit of current clinical practice.

Five fellowship-trained Consultant Radiologists read each of the 100 cases independently. The CESM examinations were anonymised and displayed on a 5 Megapixel mammography-approved workstation. Each reader had between eight and twenty-five years' breast imaging experience and fulfilled the 
NHSBSP Quality Assurance Criteria for screening mammography film reading and assessment.

Initially the LE images were read in isolation, scoring any abnormalities using the UK Royal College Of Radiologists Breast Imaging Classification scale (where 1 is normal, 2 is benign, 3 is indeterminate, 4 is suspicious and 5 is malignant (8)). At least 3 weeks later, the process was repeated, viewing both the LE and the recombined images together. Brief, anonymized, clinical information was available to the readers for both reads, for example "Patient feels lump left upper outer quadrant". The radiologists were blinded to the final outcome and diagnosis in each case.

The radiologists were asked to rate the usefulness of the recombined images in their decision-making on a 3 point scale, where $1=$ no added value, $2=$ useful aid to diagnosis, 3 = significant aid to diagnosis.

Pathology data was obtained for all women undergoing biopsy and for any subsequent surgery. Histopathology provided the definitive ground truth for those subject to biopsy.

For patients undergoing NAC, baseline MRI has remained our standard practice and therefore, in this group, tumour size was compared with reported size at baseline MRI. Breast MRI examinations were performed according to the standard local protocol on a GE 1.5T scanner, using a dedicated breast coil. Where patients had surgery as their initial therapy, size was compared with reported histopathological (whole tumour) size.

Differences in performance were assessed using receiver operative characteristic (ROC) analysis. The symptomatic lesion, as documented in the clinical information provided, was deemed to be the "index lesion" in all cases. For ROC analysis, the highest score assigned to each breast was taken as the overall opinion - for example, where two lesions were described in one breast, the more suspicious lesion was used. Sensitivity and specificity were analysed using 2-way independent t-test. Comparison of lesion size with MRI or histopathology was done using a t-test. A $p$ value of $<0.05$ was regarded as statistically significant. 
Between November 2013 and September 2014, one hundred CESM examinations were performed in ninety-nine female individuals.

98 examinations were bilateral. 1 patient underwent unilateral CESM due to a previous mastectomy. 1 patient had previously had a reaction to gadolinium at baseline MRI and so underwent unilateral CESM to assess residual tumour size post NAC in addition to the bilateral CESM examination performed at the time of diagnosis.

The mean age of the patient group at the time of CESM was 48.6 years (range $25-69$ years). The CESM examination was well tolerated by the patient group although one individual 'collapsed' during the CESM examination - this was treated initially as anaphylaxis, although subsequent investigations revealed underlying cardiac problems. The patient recovered fully.

The final diagnosis and outcome in each case is shown in Fig.1. There were 73 malignant cases and 27 with a benign final outcome. Of the malignant cases, 70 were invasive cancers, 2 were ductal carcinoma in situ and one was a papillary carcinoma. In the benign group, the commonest cause for a clinically suspicious area with subsequent benign diagnosis was a cyst $(11 / 27,41 \%)$. A case with both benign and malignant pathology is shown in figure 2 .

\section{Blind Retrospective review}

ROC analysis showed overall performance when reviewing the entire CESM study (LE and recombined images) was significantly better than LE images viewed alone (Fig.3), with area under the curve of $93 \%$ vs $83 \%(p<0.025)$.

Table 1 shows sensitivity and specificity for LE and CESM examinations. The complete sensitivity (malignant cases rated 3, 4 or 5) was improved as was the specificity (benign cases rated 1 or 2 ) when the entire CESM study was reviewed. Each of the five readers showed improved sensitivity and specificity when reviewing the entire CESM study.

MRI examinations were performed in $30 \%$ of cases (30/100). These were usually performed as a baseline prior to NAC, although four of these cases went on to have primary surgical treatment. Figures 4 and 5 show the level of agreement between CESM and the tumour size at MRI or histopathology. For 
those cases where an MRI was performed, the difference between CESM size and $\mathrm{MRI}$ size was significantly reduced compared to the LE images alone $(p<0.001)$. For the 46 cases that had primary surgical excision, there was also a significant reduction in the size difference between CESM and histological size compared to the LE images alone $(p<0.0001)$. LE images interpreted alone tended to underestimate tumour size.

The results of the readers rating of the usefulness of the recombined image in the interpretation of the CESM study is show in figure 6. Overall (using combined reader scores), the addition of the recombined images was deemed a useful aid to diagnosis in $40 \%$ cases and a significant aid to diagnosis in $35 \%$.

\section{False Negative and False Positive Cases during Clinical Use}

There were four false-negative CESM examinations in the study group. Two patients had CESM reported as normal, but the area of concern in each case was off the mammographic field (one patient had pectus excavatum). Triple assessment in both cases yielded a malignant diagnosis (both areas were palpable and also evident at ultrasound). A third patient had a CESM examination following C5 cytology taken at ultrasound. This showed no abnormality, but a $4 \mathrm{~mm}$ lobular carcinoma was found at surgical excision.

The fourth patient had a cancer diagnosed 14 months after the initial CESM examination. Low energy views were benign and recombined views showed subtle patchy linear enhancement in the area of clinical concern. A freehand core biopsy at this time was benign - B2 with inflammatory changes. The patient re-presented with increasing symptoms and a repeat CESM (not included in this review) showed florid and extensive enhancement. Core biopsy showed a high nuclear grade, well-circumscribed encapsulated papillary carcinoma-B5C.

There was one false positive CESM case. A focal area of suspicious enhancement was demonstrated at the site of clinical concern at CESM, but triple assessment including ultrasound guided core biopsy showed fat necrosis. 
In this study, the routine use of CESM as the first line mammographic modality for a symptomatic patient population with clinically suspicious abnormalities led to a significant improvement in diagnostic accuracy. Other groups have also recognised the value of CESM in cancer detection. Several studies have demonstrated consistent superiority of CESM when compared to FFDM (9) (10) (11) (12). Cheung et al (10) reported 89 women undergoing both CESM and conventional mammography. They concluded that CESM provided additional information over mammography, with consistent improvement of cancer diagnosis especially in women with a dense breast parenchymal pattern.

In our symptomatic practice, CESM consisting of the low energy image and a recombined image has replaced conventional FFDM in patients presenting with clinically suspicious abnormalities. The low energy images generated during the CESM exam have previously been shown to be equivalent to standard FFDM (3) (4). Fallenberg's group (3) compared FFDM with CESM alone and with CESM plus FFDM, looking at the detection and size estimation of histologically proven breast cancers in 118 women. CESM alone had the same sensitivity and better size assessment as CESM plus FFDM and was significantly better than FFDM alone. CESM alone also had the closest correlation with pathological size. Interestingly they found that when FFDM images where available in addition to CESM this led to a systematic overestimation of the average lesion size. They concluded that when a CESM examination is undertaken additional mammography with FFDM can be avoided. This is the approach adopted in our practice, enabling patients to benefit from the improved diagnostic accuracy of CESM, without the extra radiation dose of an additional FFDM examination. The radiation dose of CESM (to include both the LE and high energy components) is higher than FFDM, with reported increase in average glandular dose of between 6 and 81\% (3) (13) (14). However, the doses recorded in these studies still met the recommendations for maximum dose in mammography.

In the past, concern has been raised regarding potential obscuration of microcalcifications on the LE images due to the administration of contrast agent. This is an important consideration if conventional FFDM is to be omitted from the imaging pathway. It has been shown that calcifications are actually better visualised on the LE component of a CESM examination than on 
standard FFDM (15). It should be remembered that the CESM exam consists of two sets of images, both of which require equal attention - calcification may be obvious on the LE images, but not enhance on the recombined views. If calcifications are deemed suspicious on the LE views, they should be worked up in the same way as if they had been seen on standard FFDM.

$30 \%$ of the patient in our series also underwent MRI examination. This was typically performed in patients who were candidates for neo-adjuvant chemotherapy. Our local protocol continues to use MRI to monitor response to neoadjuvant chemotherapy - little work has been done to investigate a potential role for CESM in this setting. MRI can be a useful tool in staging breast cancer, particularly in patients diagnosed with lobular carcinoma or mammographically occult tumours (16). The use of MRI has the potential to delay the diagnostic process. Scanner time and availability is often an issue. If additional lesions are identified, a "second-look" ultrasound and perhaps further biopsy (either ultrasound or MR-guided) may be required. The great strength of using CESM as the first line mammographic investigation is that additional staging information and any further biopsies can be obtained at the first patient visit. In our study, CESM better reflected both MRI and histopathological size. Accuracy was much improved compared to the LE portion of the study alone which tended to underestimate tumour size. Others have also found good agreement between CESM, MRI measurements and histopathology (6) (7) (12) (17).

Since the premise of CESM is to identify abnormal enhancement within the breast, there is concern that the technique may identify lesions which are not of clinical relevance and potentially lead to "overtreatment" of disease. An increase in mastectomy rates has been attributed to the same mechanism in centres routinely performing breast MRI for staging. This concern has not been borne out in the initial clinical experience of CESM. Lobbes et al. in a study of 113 CESM examinations performed in women recalled from the Dutch screening programme found that MRI detected more ipsilateral additional foci of tumour cells in women diagnosed with a breast cancer ( $88 \%$ of additional foci were detected using MRI compared to 56\% with CESM) (7). However, CESM resulted in fewer false positives - 13 false positives were observed at MRI, compared to only two false-positives using CESM. The number of multifocal tumours undergoing MRI in our series was too small to make any 
meaningful comparison with CESM. There was one false positive case in our own series with a focal area of suspicious enhancement demonstrated at the site of clinical concern on CESM, with percutaneous biopsy demonstrating fat necrosis. As with MRI it is important that the nature of abnormal areas of enhancement is determined with percutaneous biopsy before treatment decisions are made. Further work is still needed before CESM can be recommended as a replacement for MRI.

Clearly CESM takes longer to perform than a standard mammogram. Most of this additional time is taken in patient consent, excluding contraindications and establishing intravenous access. We estimate that this adds approximately 1015 minutes per exam. These tasks could be delegated to mammography staff with appropriate training. Once the patient is in the room and the injection has been given, the mammographic technique is identical to standard FFDM. The images can be reviewed immediately and any further areas of concern can be investigated during the same clinic appointment.

The administration of iodinated contrast agents is not completely without risk. We do not administer contrast agent to patients who have or at risk of renal impairment. The potential for contrast agent toxicity needs to be considered. All staff involved in contrast administration have had the appropriate training to recognise and treat contrast agent reactions, with the necessary drugs and resuscitation equipment immediately available. We had one potential contrast agent reaction in this series of our first 100 cases, but on further investigation this may well have been cardiac in origin rather than true anaphylaxis.

The CESM technique is generally well-accepted by staff and patients. Hobbs et al (18) looked at the patient experience of CESM compared with MRI during preoperative breast cancer staging. A significantly higher overall preference towards CESM was demonstrated, with patients citing faster procedure time, greater comfort and lower noise levels. Participants also reported lower rates of anxiety during CESM compared with CEMRI. In our study, the reporting radiologists also found CESM to be a useful tool. In 75\% of cases CESM was deemed a useful or significant aid to diagnosis.

There are limitations to this study. The majority of patients undergoing CESM had a clinically suspicious abnormality; consequently the case-mix is skewed towards larger tumours. Research suggests that sizing of larger tumours is less accurate, with CESM tending to oversize compared to MRI (7) (19). Consequently it may not be possible to extrapolate the findings to other 
patient groups were tumours may be smaller. Further work is needed in other symptomatic and screening populations. In addition, although this multireader study was blinded, the readers knew that the patients were symptomatic, and were also aware of the site of clinical concern. We have a taken a pragmatic approach and although this may result in bias the CESM study was interpreted as it would be in clinical practice.

In conclusion, CESM provides immediately available, clinically useful information in the symptomatic clinic in patients with suspicious palpable abnormalities. Radiologist sensitivity, specificity and size accuracy for breast cancer detection and staging are all improved using CESM as the primary mammographic investigation. The routine use of the technique in other settings still requires further evaluation. 
Fig.1 Final diagnosis and outcome

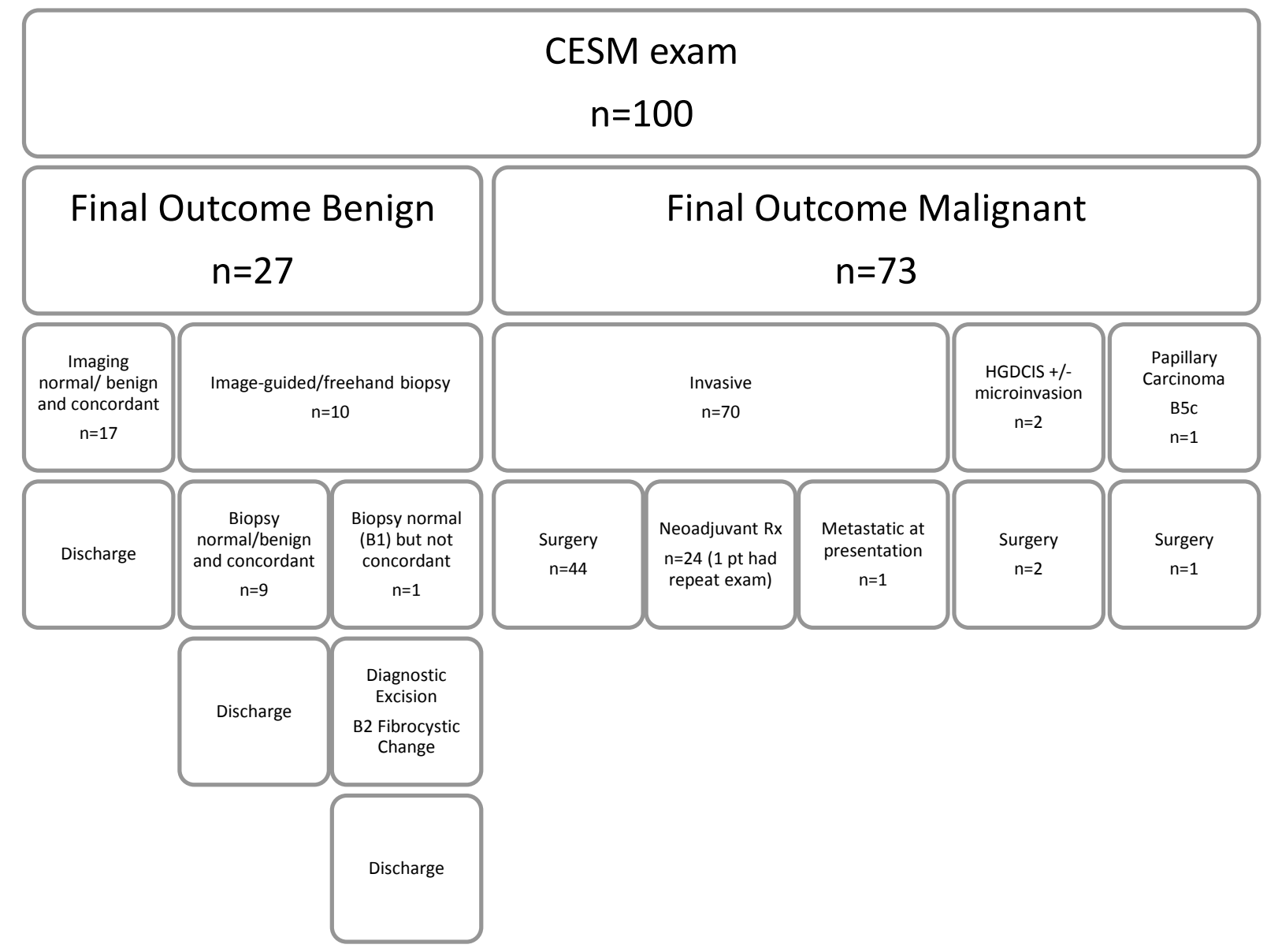


Table 1 Individual Reader Performance

\begin{tabular}{|c|c|c|c|c|}
\hline & \multicolumn{2}{|c|}{ Complete Sensitivity $(\mathrm{M} 3,4,5)$} & \multicolumn{2}{|c|}{ Specificity } \\
\hline & LE & CESM & LE & CESM \\
\hline R1 & $89.0 \%$ & $93.2 \%$ & $70.4 \%$ & $74.1 \%$ \\
\hline R2 & $84.9 \%$ & $91.8 \%$ & $70.4 \%$ & $85.2 \%$ \\
\hline R3 & $86.3 \%$ & $94.5 \%$ & $51.9 \%$ & $81.5 \%$ \\
\hline R4 & $84.9 \%$ & $95.9 \%$ & $59.3 \%$ & $81.5 \%$ \\
\hline R5 & $76.7 \%$ & $97.3 \%$ & $63.0 \%$ & $81.5 \%$ \\
\hline Mean & $84.4 \%$ & $94.5 \%$ & $63.0 \%$ & $80.7 \%$ \\
\hline$p$ (t-test) & \multicolumn{2}{|c|}{0.023} & \multicolumn{2}{|c|}{0.014} \\
\hline
\end{tabular}

Fig.3 Overall Performance of CESM vs LE alone

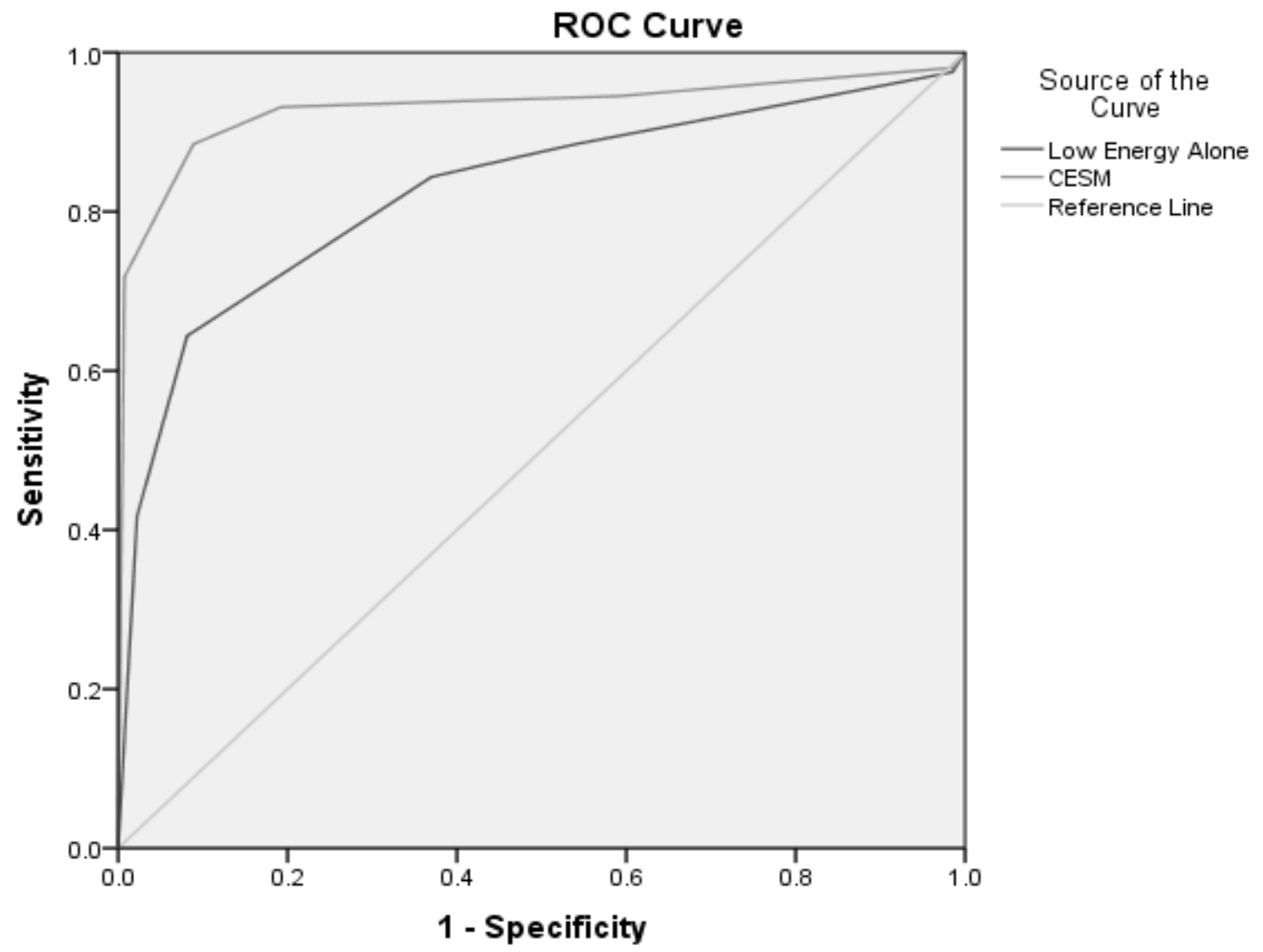

Diagonal segments are produced by ties. 
Fig.4 Size agreement between CESM and reported tumour size at MRI

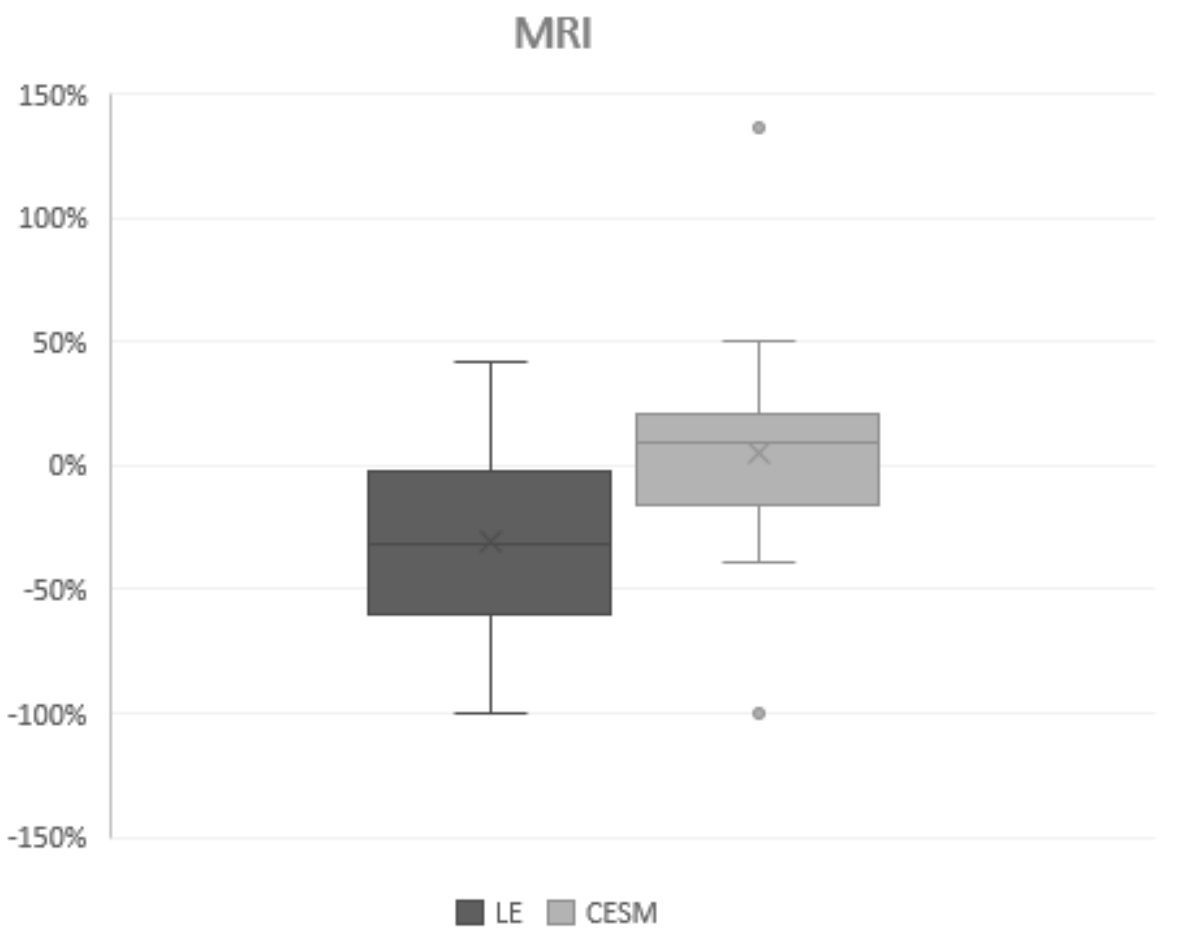

Fig.5 Size agreement between CESM and histopathology (whole tumour size)

Histopathology

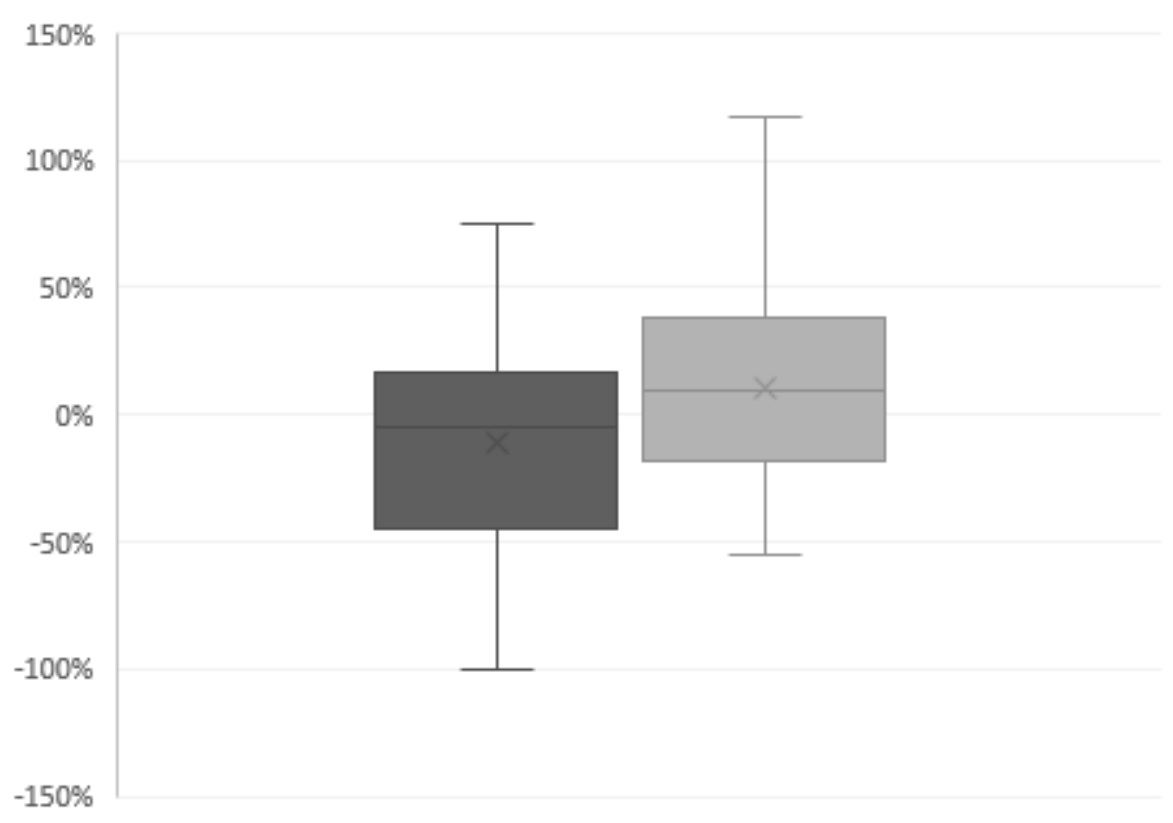

$\square$ LE $\square$ CESM 
Fig.6 Reader-perceived usefulness of recombined views

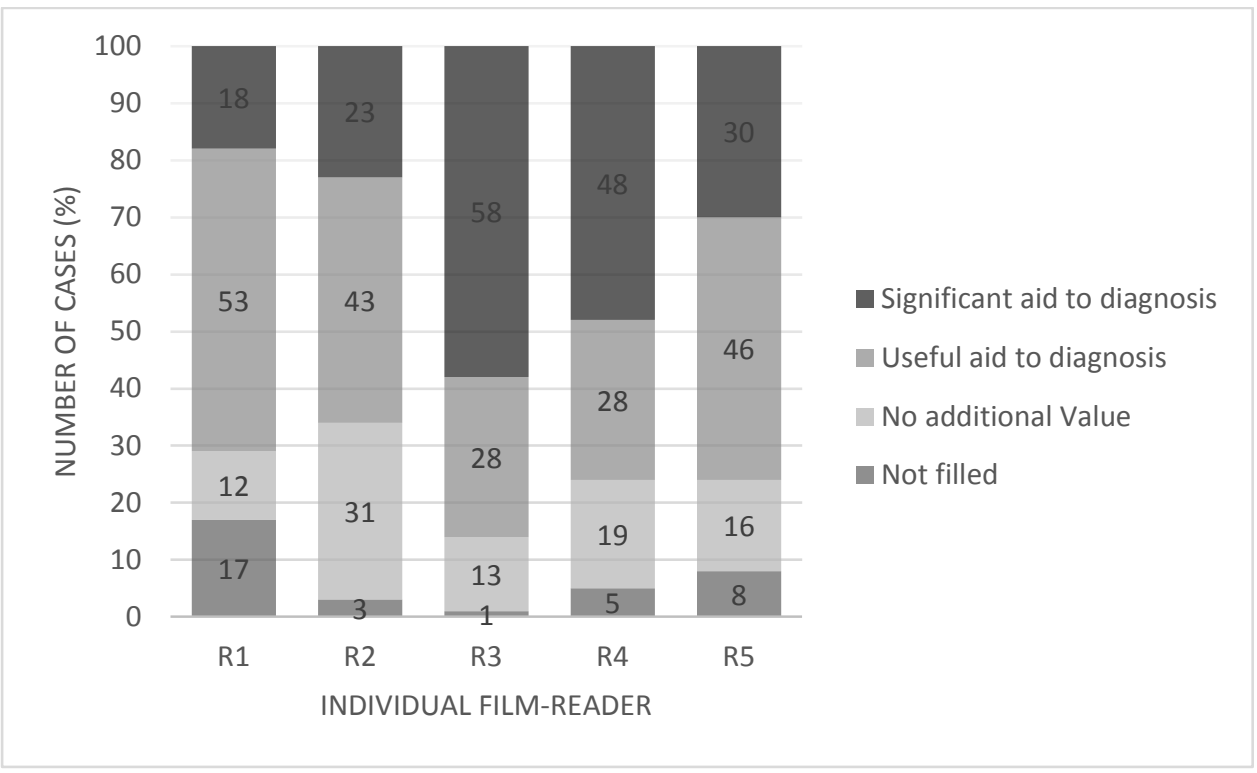


Fig. 2

A 46 year old female presented to clinic, having noticed lumps in both breasts. Clinical examination revealed a suspicious mass in the left breast (P5) and an indeterminate mass in the right breast (P3).

Contrast mammography was performed. LE images (A-D) show a dense background pattern. There is a mass in the upper inner aspect of the right breast and some subtle distortion in the upper outer quadrant of the left breast.

Recombined images (E-H) demonstrate the "eclipse" sign of a cyst within the right breast. This was confirmed at ultrasound. A large area of enhancement is seen in the upper left breast - at least $50 \mathrm{~mm}$. Ultrasound core biopsy proved grade 2 tumour with lobular features.

MRI pre-NAC (I) showed approximately $60 \mathrm{~mm}$ of enhancement within the left breast. The right breast was benign at MRI.
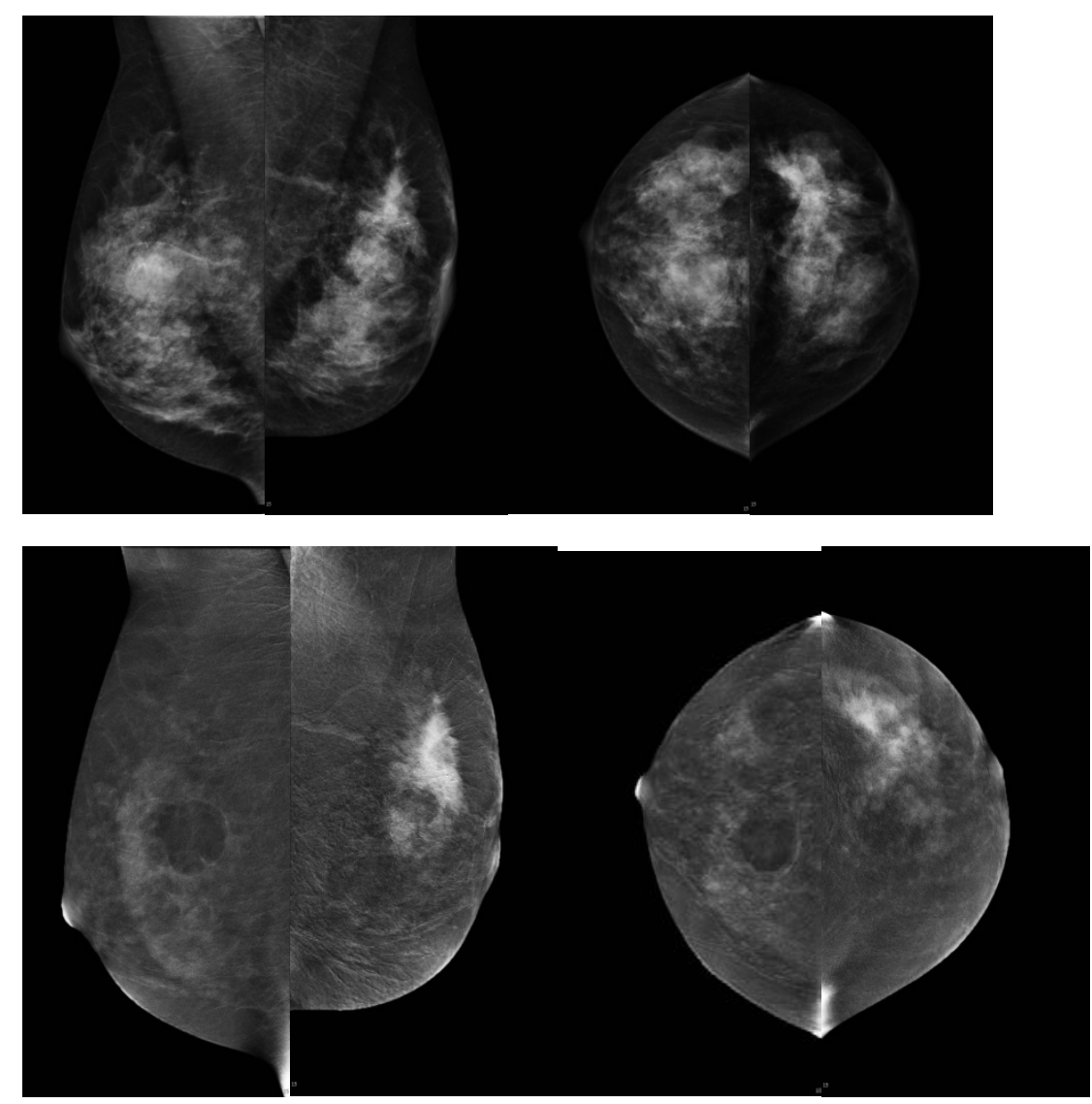


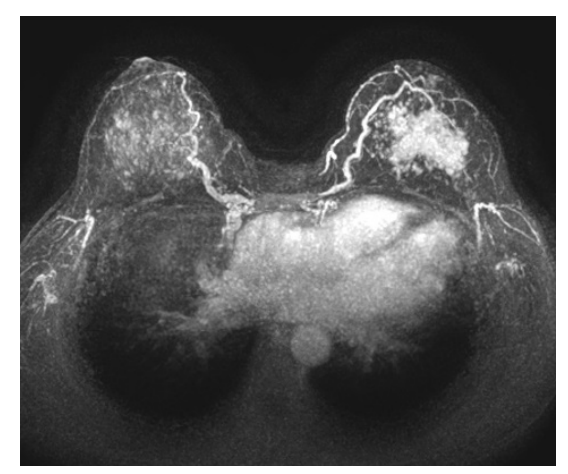


1. Best practice diagnostic guidelines for patients presenting with breast symptoms. Willett $A M$ et al, 20103. .

2. Individual and combined effects of age, breast density and hormone replacement use on the accuracy of screening mammography. Carney PA, Miglioretti DL. 2003, Annals of Internal Medicine, p. 168.

3. Contrast-enhanced spectral mammography: Does mammography provide additional clinical benefits or can some radiation exposure be avoided? . Fallenberg EM, Dromain C, Diekmann F, Renz DM, Amer H, Ingold-Heppner B, Neumann AU, Winzer KJ, Bick U, Hamm B, Engelken F. Breast Cancer Res Treat. 2014 Jul;146(2):371-81.doi: 10.1007/s10549-014-3023-6. Epub 2014 Jul 2. PubMed PMID: 24986697.

4. Low energy mammogram obtained in contrast-enhanced digital mammography (CEDM) is comparable to routine full-field digital mammography (FFDM). al., Francescone MA et. 2014, Eur J Radiol. 2014 Aug; 83(8):1350-5. .

5. Eur Radiol. 2015 Oct;25(10):2813-20. doi: 10.1007/s00330-015-3695-2. Epub 2015 Mar 27. PubMed PMID: 25813015; PubMed Central PMCID: PMC4562003. Lalji UC, Jeukens CR, Houben I, Nelemans PJ, van Engen RE, van Wylick E, Beets-Tan RG, Wildberger JE, Paulis LE, Lobbes MB. Evaluation of low-energy contrast-enhanced spectral mammography images by comparing them to full-field digital mammography us.

6. Contrast-enhanced spectral mammography versus MRI: Initial results in the detection of breast cancer and assessment of tumour size. Fallenberg EM, Dromain C, Diekmann F, Engelken F, Krohn M, Singh JM, Ingold-Heppner B, Winzer KJ, Bick U, Renz DM. Eur Radiol. 2014 Jan;24(1):256-64. doi: 10.1007/s00330-013-3007-7. Epub 2013 Sep 19. PubMed PMID: 24048724.

7. Contrast-enhanced spectral mammography in patients referred from the breast cancer screening programme. Lobbes MB, Lalji U, Houwers J, Nijssen EC, Nelemans PJ, van Roozendaal L, Smidt ML, Heuts E, Wildberger JE. Eur Radiol. 2014 Jul;24(7):1668-76. doi: 10.1007/s00330-014-3154-5. Epub 2014 Apr 3. PubMed PMID: 24696228.

8. The Royal College of Radiologists Breast Group breast imaging classification. AJ, Maxwell. 2009, Clin Radiol., pp. 64(6):624-7.

9. Contrast enhanced mammography: Techniques, current results, and potential indications. M.B.I. Lobbes, M.L. Smidt, J. Houwers, V.C. Tjan-Heijnen, J.E. Wildberger. Issue 9 p935-944, s.I. : Clinical Radiology, 2013, Vol. Vol. 68.

10. Diagnostic performance of dual-energy contrast-enhanced subtracted mammography in dense breasts compared to mammography alone: interobserver blind-reading analysis. Cheung YC, Lin YC, Wan YL, Yeow KM, Huang PC, Lo YF, Tsai HP, Ueng SH, Chang CJ. s.I. : Eur Radiol , 2014, Eur Radiol. 2014 Oct;24(10):2394-403. doi: 10.1007/s00330-014-3271-1. Epub 2014 Jun 14. PubMed PMID: 24928280., Vols. 24:2394-2403 .

11. Luczyńska E, Heinze-Paluchowska S, Dyczek S, Blecharz P, Rys J, Reinfuss M.Contrast-enhanced spectral mammography: comparison with conventional mammography and histopathology in 152 women. Korean J Radiol. 2014 Nov-Dec and doi:10.3348/kjr.2014.1, 15(6):689-96. 
12. Bilateral Contrast-enhanced Dual-Energy Digital Mammography: Feasibility and Comparison with Conventional Digital Mammography and MR Imaging in Women with Known Breast Carcinoma. Maxine S. Jochelson, MD, D. David Dershaw, MD, Janice S. Sung, MD, Alexandra S. Heerdt, MD, Cynthia Thornton, RT(R)(M), Chaya S. Moskowitz, MD, Jessica Ferrara, BS, and Elizabeth A. Morris, MD. 3, s.I. : Radiology, March 2013, Vol. 266. DOI: http://dx.doi.org/10.1148/radiol.12121084.

13. Dual-energy contrast enhanced digital mammography:initial clinical results of a multireader, multicase study. Dromain C, Thibault F, Diekmann F, Fallenberg E, Jong R et al. s.l. : Breast Cancer Research, 2012, Vol. 14:R94.

14. Jeukens CR, Lalji UC, Meijer E, Bakija B, Theunissen R, Wildberger JE, Lobbes MB. Radiation exposure of contrast-enhanced spectral mammography compared with full-field digital mammography. Invest Radiol. 2014 Oct and doi:10.1097/RLI.00000000000, 49(10):659-65.

15. Clinical Utility of dual-energy contrast-enhanced spectral mammography for breast microcalcifications without associated mass: a preliminary analysis. Cheung YC, Tsai HP, Lo YF, Ueng SH, Huang PC, Chen SC. July 10, s.l. : Eur J Radiol, 2015.

16. Early and locally advanced breast cancer: diagnosis and treatment (CG80). NICE. s.I. : National Institute for Health and Care Excellence, 2009.

17. The quality of tumor size assessment by contrast-enhanced spectral mammography and the benefit of additional breast MRI. . Lobbes MB, Lalji UC, Nelemans PJ, Houben I, Smidt ML, Heuts E, de Vries B, Wildberger JE, Beets-Tan RG. s.I. : J Cancer. Jan 5; 6(2):144-, 2015.

18. Hobbs MM, Taylor DB, Buzynski S, Peake RE. Contrast-enhanced spectral mammography (CESM) and contrast enhanced MRI (CEMRI): Patient preferences and tolerance. J Med Imaging Radiat Oncol. 2015 Jun and 21, 59(3):300-5. doi: 10.1111/1754-9485.12296. Epub 2015 Apr.

19. Use of Contrast-Enhanced Spectral Mammography for Intramammary Cancer Staging: Preliminary Results. Blum K S, Rubbert C, Mathys B, Antoch G et al. 11, s.I. : Academic Radiology, 2014, Vol. 21. 